International Journal of Pure and Applied Mathematics

Volume 87 No. 1 2013, 181-194

ISSN: 1311-8080 (printed version); ISSN: 1314-3395 (on-line version)

url: http://www.ijpam.eu

doi: http://dx.doi.org/10.12732/ijpam.v87i1.11

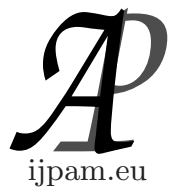

\title{
JACOBI STABILITY OF HAMILTONIAN SYSTEMS
}

\author{
Hossein Abolghasem \\ Department of Information Systems \\ St. Francis Xavier University \\ Antigonish, Nova Scotia, B2G 2W5, CANADA
}

\begin{abstract}
The stability of Hamiltonian systems with one degree of freedom is investigated from Jacobi stability point of view (the KCC theory) and it is shown that the Jacobi stability conditions of non-degenerate equilibrium points are the same as known conditions for Liapunov stability.
\end{abstract}

AMS Subject Classification: 34D20, 34D35, 34C05, 37C10, 70K20, 70E50, 70K05, 70H05, 37Jxx

Key Words: dynamical systems, Liapunov stability, Jacobi stability, KCC theory, Hamiltonian systems

\section{Introduction}

The Jacobi stability is a natural generalization of the stability of the geodesic flow on a differentiable manifold equipped with a Riemannian or Finslerian metrics to the non-metric manifold. The Jacobi (also called KCC) stability examines the robustness of a second-order differential equation, where robustness is a measure of insensitivity and adaptation to change of the system internal parameters and the environment. Jacobi stability analysis of dynamical systems has been recently studied by several authors $[6,9,11,17,18,19]$ using the Kosambi-Cartan-Chern (KCC) theory.

From the mathematical point of view, the KCC theory investigates the deviation of neighboring trajectories, which enables one to estimate the perturbation permitted around the steady states solutions of the second-order differential equation. This is of interest in physical applications when one needs to identify

Received: June 20, 2013

(c) 2013 Academic Publications, Ltd. url: www.acadpubl.eu 
the "robust arrest" regions, i.e., the regions where one has both Liapunov and Jacobi stability.

The KCC theory originated from the works of D. D. Kosambi [14], E. Cartan [12] and S. S. Chern [13], and hence the abbreviation KCC (Kosambi-CartanChern). The KCC theory has applications in engineering, physics, chemistry and biology. Recent developments have been made in KCC applications in gravitation and cosmology. Boehmer et al in [19] have analyzed Jacobi stability and its relations with the linear Liapunov stability analysis of dynamical systems, and presented a comparative study of these methods in the fields of gravitation and astrophysics. What has been demonstrated in their work is that there are cases investigated where Liapunov stability is not in agreement with Jacobi stability. Most recently $[1,2,3]$ demonstrated that these two types of stability concepts are in agreement in three cases of torque-free rigid body motion around a stationary point, circular orbits in a central force field, and circular orbits in Schwarzschild spacetime.

The purpose of the present paper is to study the stability of non-degenerate equilibrium points of a Hamiltonian system with degree of freedom and show that Liapunov stability is entirely in agreement with Jacobi stability. We review the KCC theory to analyze its relations with the linear Liapunov stability analysis of dynamical systems, and to present a comparative study of these methods for a Hamiltonian system with one degree of freedom.

The present paper is organized as follows. In Section 2 we briefly review the classical Liapunov stability analysis. Section 3 is devoted to the KCC theory and the Jacobi stability analysis of the planar dynamical systems. A very brief introduction to Hamiltonian systems with one degree of freedom is presented in Section 4. A full dynamical systems approach leading to stability analysis of (Liapunov and Jacobi) of non-degenerate equilibrium points are given in Section 5. A discussion and final remarks appears in Section 6.

\section{Liapunov Stability}

\subsection{Brief Survey of Techniques in Dynamical Systems}

In this section we give precise mathematical expression to the concepts of stability of dynamical systems used in this research [15, 16, 21].

Definition. A fixed point of a system of autonomous ODEs

$$
\dot{\mathbf{x}}=\mathbf{f}(\mathbf{x})
$$


is a point $\overline{\mathbf{x}} \in \mathbf{R}^{n}$ such that $\mathbf{f}(\overline{\mathbf{x}})=0$, where $\mathbf{f}$ is a $C^{1}$ vector field in $\mathbf{R}^{n}$. In the present paper, the fixed points are also called equilibrium point interchangeably. We reserve the term critical point for referring to extrema and saddle of a 2dimensional surface.

Definition. Let $\overline{\mathbf{x}}$ be a fixed point of the DE (1). The point $\overline{\mathbf{x}}$ is called a non-degenerate fixed point if $\lambda_{i} \neq 0$ for all eigenvalues, $\lambda_{i}$, of the Jacobian of the vector field $\mathbf{f}(\mathbf{x})$ evaluated at $\overline{\mathbf{x}}$. Otherwise the point is called degenerate.

Definition. Let $\overline{\mathbf{x}}$ be a fixed point of the DE (1). The point $\overline{\mathbf{x}}$ is called a hyperbolic fixed point if $\operatorname{Re}\left(\lambda_{i}\right) \neq 0$ for all eigenvalues, $\lambda_{i}$, of the Jacobian of the vector field $\mathbf{f}(\mathbf{x})$ evaluated at $\overline{\mathbf{x}}$. Otherwise the point is called non-hyperbolic.

Definition. (Liapunov Stability) $\overline{\mathbf{x}}(t)$ is said to be stable (or Liapunov stable) if, given $\epsilon>0$, there exists a $\delta=\delta(\epsilon)>0$ such that, for any other solution, $\mathbf{y}(t)$, of (1) satisfying $\left|\overline{\mathbf{x}}\left(t_{0}\right)-\mathbf{y}\left(t_{0}\right)\right|<\delta$, then $|\overline{\mathbf{x}}(t)-\mathbf{y}(t)|<\epsilon$ for $t>t_{0}, t_{0} \in \mathbf{R}$. A solution which is not stable is said to be unstable.

Definition. (Asymptotic Stability) $\overline{\mathbf{x}}(t)$ is said to be asymptotically stable if it is Liapunov stable and for any other solution, $\mathbf{y}(t)$, of $(1)$, there exists a constant $b>0$ such that, if $\left|\overline{\mathbf{x}}\left(t_{0}\right)-\mathbf{y}\left(t_{0}\right)\right|<b$, then $\lim |\overline{\mathbf{x}}(t)-\mathbf{y}(t)|=0$ as $t \rightarrow \infty$.

One must begin a qualitative analysis of a dynamical system by locating its fixed points. Once the fixed points are obtained, one should consider the dynamics in a local neighborhood of each of the points. Assuming that the vector field $\mathbf{f}(\mathbf{x})$ is of class $C^{1}$, then the local behavior of the orbits can be determined by linear approximation of the vector field in the local neighborhood of the fixed point $\overline{\mathbf{x}}$. In this neighborhood,

$$
\mathbf{f}(\mathbf{x}) \approx D \mathbf{f}(\overline{\mathbf{x}})(\mathbf{x}-\overline{\mathbf{x}}),
$$

where $D \mathbf{f}(\overline{\mathbf{x}})$ is the Jacobian of the vector field at the fixed point $\overline{\mathbf{x}}$. The system (2) is referred to as the linearization of the DE at the fixed point. The classification of the fixed points can then be done by studying the eigenvalues of the Jacobian of the linearized vector field at the point. The classification then follows from the fact that if the fixed point is hyperbolic in nature the orbits of the non-linear system and its linear approximation are topologically equivalent in a neighborhood of the fixed point. The following important theorem supports this fact:

Theorem 1. (Hartman-Grobman Theorem) Consider a DE $\dot{\mathbf{x}}=\mathbf{f}(\mathbf{x})$, $x \in \mathbf{R}^{n}$ where the vector field $\mathbf{f}$ is of class $C^{1}$. If $\overline{\mathbf{x}}$ is a hyperbolic fixed point of 
the DE then there exists a neighborhood of $\overline{\mathbf{x}}$ on which the flow is topologically equivalent to the flow of the linearization of the $D E$ at $\overline{\mathbf{x}}$.

Given a linear system of ODEs:

$$
\dot{\mathbf{x}}=\mathbf{A x}
$$

where $A$ is a $n \times n$ square matrix with constant coefficients, it is a straightforward to show when eigenvalues of $A$ have negative real parts, then the fixed point is a hyperbolic sink (stable), since all solutions converge to the fixed point $\overline{\mathbf{x}}=0$; a linear system whose eigenvalues have positive real parts is called to have a hyperbolic source (unstable), because the solutions in the neighborhood of $\overline{\mathbf{x}}=0$ all diverge from that point; and when one eigenvalue is negative and the other is positive the fixed point is said to be a hyperbolic saddle (unstable), where some orbits are attracted to the fixed point, and some are repelled away. If the eigenvalues are complex conjugates with non-zero real parts then the fixed point is of type spiral (stable or unstable depends on the sign of $\operatorname{Re}\left(\lambda_{i}\right)$ ) and if $\operatorname{Re}\left(\lambda_{i}\right)=0$, then the fixed point is of type center.

In the non-linear case, the topological equivalence of flows allows for a similar classification of the fixed points. The equivalence only applies in directions where the eigenvalue has non-zero real parts. If the linearization of the dynamical systems at the fixed point has purely imaginary eigenvalues, then the stability type of equilibrium point cannot be deduced from the linear approximation. In such case one must use other methods such as Liapunov stability analysis via constructing a Liapunov function to analyze the case. For the case of 2-dimensional systems, the following theorem is useful for our upcoming analysis:

Theorem 2. Let $E$ be an open subset of $\mathbf{R}^{2}$ containing the origin and let $\mathbf{f}$ be analytic in $E$ with $\mathbf{f}(\mathbf{0})=\mathbf{0}$. Suppose that the origin is a center for the linear system (3) with $A=D \mathbf{f}(\mathbf{0})$. Then the origin is either a center or a focus for the nonlinear system (1).

This brief introduction concludes our treatment of Liapunov stability of dynamical systems which is used in this paper.

\section{Kosambi-Cartan-Chern (KCC) Theory and Jacobi Stability}

\subsection{General Theory}

We now introduce the main ideas of $\operatorname{KCC}$-theory $[5,6,9,11]$. Let $\mathcal{M}$ be a real, smooth $n$-dimensional manifold and let $T \mathcal{M}$ be its tangent bundle. Let 
$\mathbf{u}=(\mathbf{x}, \mathbf{y})$ be a point in $T \mathcal{M}$, where $\mathbf{x}=\left(x^{1}, x^{2}, \ldots, x^{n}\right)$, and $\mathbf{y}=\left(y^{1}, y^{2}, \ldots, y^{n}\right)$ which means $y^{i}=\frac{d x^{\mathrm{i}}}{d t}, i=1,2, . ., n$. Consider the second order differential equations in normalized form

$$
\frac{d^{2} x^{i}}{d t^{2}}+2 G^{i}(\mathbf{x}, \mathbf{y})=0, \quad i=1,2, \ldots, n,
$$

where $G^{i}(\mathbf{x}, \mathbf{y})$ are smooth functions defined in a local system of coordinates on $T \mathcal{M}$. In fact the system of (4) is motivated by Euler-Lagrange equations of classical dynamics

$$
\begin{array}{ll}
\frac{d}{d t} \frac{\partial L}{\partial y^{i}}-\frac{\partial L}{\partial x^{i}}=F_{i} & i=1,2, \ldots, n, \\
y^{i}=\frac{d x^{i}}{d t}, &
\end{array}
$$

where $L$ is the Lagrangian of $\mathcal{M}$, and $F_{i}$ are the external forces [8].

In order to find the KCC differential invariants of the system (4) under the non-fixed coordinate transformation

$$
\begin{aligned}
& \tilde{x}^{i}=\tilde{x}^{i}\left(x^{1}, x^{2}, \ldots, x^{n}\right) \\
& \tilde{y}^{i}=\frac{\partial \tilde{x}^{i}}{\partial x^{j}} y^{j}
\end{aligned} \quad i=1,2, \ldots, n .
$$

We now define the $K C C$-covariant differential of a vector field $\xi=\xi^{i}(x) \frac{\partial}{\partial x^{\top}}$ in an open subset $\Omega \subseteq \mathbf{R}^{n} \times \mathbf{R}^{n}$ as $[5,6,9,19]$

$$
\frac{D \xi^{i}}{d t}=\frac{d \xi^{i}}{d t}+N_{j}^{i} \xi^{j}
$$

where $N_{j}^{i}=\frac{\partial G^{\mathrm{i}}}{\partial y^{j}}$ defines the coefficients of a non-linear connection $N$ on the tangent bundle $T \mathcal{M}$. For $\xi^{i}=y^{i}$, one obtains

$$
\frac{D y^{i}}{d t}=N_{j}^{j} y^{j}-2 G^{i}=-\epsilon^{i} .
$$

The contravariant vector field $\epsilon^{i}$ on $\Omega$ is called the first KCC invariant and plays the role of an external force. The terms $\epsilon^{i}$ has geometrical character since with respect to a coordinate transformation (6), we have

$$
\tilde{\varepsilon}^{i}=\frac{\partial \tilde{x}^{i}}{\partial x^{j}} \varepsilon^{j} .
$$


Furthermore, if one varies the paths $x^{i}(t)$ of the system (4) into nearby ones prescribed by

$$
\tilde{x}^{i}(t)=x^{i}(t)+\eta \xi^{i}(t),
$$

where $|\eta|$ is a small parameter and $\xi^{i}(t)$ are the components of some contravariant vector field defined along the path $x^{i}(t)$. We derive the following variational equations after substituting Eqs.(10) into Eqs.(4) and taking the limit as $\eta \rightarrow 0$ $[4,5,17]$

$$
\frac{d^{2} \xi^{i}}{d t^{2}}+2 N_{j}^{i} \frac{d \xi^{j}}{d t}+2 \frac{\partial G^{i}}{\partial x^{j}} \xi^{j}=0 .
$$

We can write Eqs.(11) in terms of the KCC covariant differential (7) in the covariant form

$$
\frac{D^{2} \xi^{i}}{d t^{2}}=P_{j}^{i} \xi^{j}
$$

where the right hand side $(1,1)$-tensor

$$
P_{j}^{i}=-2 \frac{\partial G^{i}}{\partial x^{j}}-2 G^{l} G_{j l}^{i}+y^{l} \frac{\partial N_{j}^{i}}{\partial x^{l}}+N_{l}^{i} N_{j}^{l},
$$

and $G_{j l}^{i} \equiv \partial N_{j}^{i} / \partial y^{l}$ is called the Berwald connection [4, 17]. Eq. (12) is called the Jacobi equations, or the variation equations associated with the system of second order differential equations (4), and $P_{j}^{i}$ is called the second $K C C$ invariant.

The third, fourth and fifth invariants of the system (4) are given by [7]

$$
P_{j k}^{i} \equiv \frac{1}{3}\left(\frac{\partial P_{j}^{i}}{\partial y^{k}}-\frac{\partial P_{k}^{i}}{\partial y^{j}}\right), \quad P_{j k l}^{i} \equiv \frac{\partial P_{j k}^{i}}{\partial y^{l}}, \quad D_{j k l}^{i} \equiv \frac{\partial G_{j k}^{i}}{\partial y^{l}}
$$

The third invariant represents a torsion tensor, while the fourth and fifth invariants are interpreted as the Riemann-Christoffel curvature tensor, and the Douglas tensor, respectively $[5,17]$. These tensors which describe the geometrical properties of a system of second-order differential equations always exist $[5,17]$.

The term Jacobi stability within the KCC theory is justified by the fact that, when (4) represents the second order differential equations for the geodesic equations in Finsler or Riemannian geometry [10], then (12) is the Jacobi field equations for the geodesic deviation. The Jacobi equation (12) of the Finsler manifold $(M ; F)$ can be written in the scalar form [10]:

$$
\frac{d^{2} v}{d s^{2}}+K \cdot v=0
$$


where $\xi^{i}=v(s) \eta^{i}$ is a Jacobi field along the geodesic $x^{i}(s), \eta^{i}$ is the unit normal vector field, and $K$ is the flag curvature of $(M ; F)$. The sign of $K$ affects the geodesic rays: if $K>0$, then the geodesics bunch together (are Jacobi stable), and if $K<0$, then they disperse (are Jacobi unstable). Therefore, positive/negative flag curvature is equivalent to negative/positive eigenvalues of $P_{j}^{i}$.

The following result is known [19] :

Theorem 3. The trajectories of (4) are Jacobi stable if and only if the real parts of the eigenvalues of the deviation tensor $P_{j}^{i}$ are strictly negative everywhere, and Jacobi unstable, otherwise.

The geometrical meaning of the above theorem in the context of an Euclidean, Riemannian or Finslerian structure is discussed in [19].

\subsection{The Relation between Linear Stability and Jacobi Stability}

The correlation between linear stability and Jacobi stability has been completely discussed in [19] for the 2-dimensional dynamical systems case. The result of this discussion is crucial for the present paper. Here one wants to compare the signs of the eigenvalues of the Jacobian matrix $J$ at a fixed point with the signs of the eigenvalues of the deviation curvature tensor $P_{i}^{j}$ evaluated at the same point.

Let us consider the following autonomous system of ODE:

$$
\left\{\begin{array}{l}
\dot{u}=f(u, v) \\
\dot{v}=g(u, v)
\end{array},\right.
$$

such that the point $(0,0)$ is an equilibrium, i.e., $f(0,0)=g(0,0)=0$. One can always make variable changes $\bar{u}=u-u_{0}$ and $\bar{v}=v-v_{0}$ to translate a general fixed point $\left(u_{0}, v_{0}\right)$ to the origin $(0,0)$. We denote by $J$ the Jacobian matrix of (15), i.e.

$$
J(u, v)=\left(\begin{array}{ll}
f_{u} & f_{v} \\
g_{u} & g_{v}
\end{array}\right),
$$

where the subscripts are partial derivatives with respect to $u$ and $v$. The characteristic equation is given by

$$
\lambda^{2}-(\operatorname{tr} A) \lambda+\operatorname{det} A=0,
$$

where $\lambda, \operatorname{tr} A$ and $\operatorname{det} A$ are the eigenvalue, the trace and the determinant of the matrix $A:=\left.J\right|_{(0,0)}$, respectively. The signs of the trace, determinant of $A$, and 
of the discriminant $\Delta=\left(f_{u}-g_{v}\right)^{2}+4 f_{v} g_{u}=(\operatorname{tr} A)^{2}-4 \operatorname{det} A$ give the linear stability of the fixed point $(0,0)$ as described in the previous section.

In applying the KCC theory to the ODE system (15), by elimination of one of the variables, one can transform (15) into a system of differential equations of the form (4) and compute the deviation curvature. Boehmer et al in [19] obtain the following theorem:

Theorem 4. Let us consider the ODE (15) with the fixed point $P=(0,0)$ such that $\left.g_{u}\right|_{(0,0)} \neq 0$. Then,

$$
\left.4 P_{1}^{1}\right|_{(0,0)}=-\left.4 g_{, 1}^{1}\right|_{(0,0)}+\left.\left(g_{, 1}^{1}\right)^{2}\right|_{(0,0)}=\Delta:=(\operatorname{tr} A)^{2}-4 \operatorname{det} A .
$$

where $g^{1}(x, y) \equiv-g_{u}(u(x, y), x) f(u(x, y), x)-g_{v}(u(x, y), x) y$ and $y \equiv g(u, v)$ and $g_{, 1}^{1}$ is simply $\frac{\partial g^{1}}{\partial x}$. In this case, the trajectory $v=v(t)$ is Jacobi stable if and only if $\Delta<0$.

More generally, we can conclude that in the case $\left.g_{u}\right|_{(0,0)} \neq 0$ and $\left.f_{v}\right|_{(0,0)} \neq 0$ both trajectories $u=u(t)$ and $v=v(t)$ are Jacobi stable if and only if $\Delta<0$. We emphasis on this theorem greatly because our main Jacobi analysis in this paper is based on this theorem. Boehmer et al in [19] also present the following corollary:

Corollary. Let us consider the ODE (15) with the fixed point $P=(0,0)$ such that $\left.g_{u}\right|_{(0,0)} \neq 0$. Then, the Jacobian $J$ estimated at the point $P$ has complex eigenvalues if and only if $P$ is a Jacobi stable point using Theorem 3 above.

A combination of the linear stability as depicted in the Fig. 1 [16], with the definition of Jacobi stability with the conditions stated in the theorem above, one obtains the following relations:

\section{Region I}

$$
\begin{array}{cc}
\Delta>0 & \text { Jacobi unstable } \\
\operatorname{tr} A>0 & \text { Unstable node } \\
\operatorname{det} A>0 &
\end{array}
$$

\section{Region II}

$\begin{array}{cc}\Delta<0 & \text { Jacobi stable } \\ \operatorname{tr} A>0 & \text { Unstable focus } \\ \operatorname{det} A>0 & \end{array}$




\section{Region III}

$\begin{array}{cc}\Delta<0 & \text { Jacobi stable } \\ \operatorname{tr} A<0 & \text { Stable focus } \\ \operatorname{det} A>0 & \end{array}$

4. Region IV

$\begin{array}{cc}\Delta>0 & \text { Jacobi unstable } \\ \operatorname{tr} A<0 & \text { Stable node } \\ \operatorname{det} A>0 & \end{array}$

\section{Region V}

$$
\begin{array}{cc}
\Delta>0 & \text { Jacobi unstable } \\
\operatorname{det} A<0 & \text { Saddle point }
\end{array}
$$

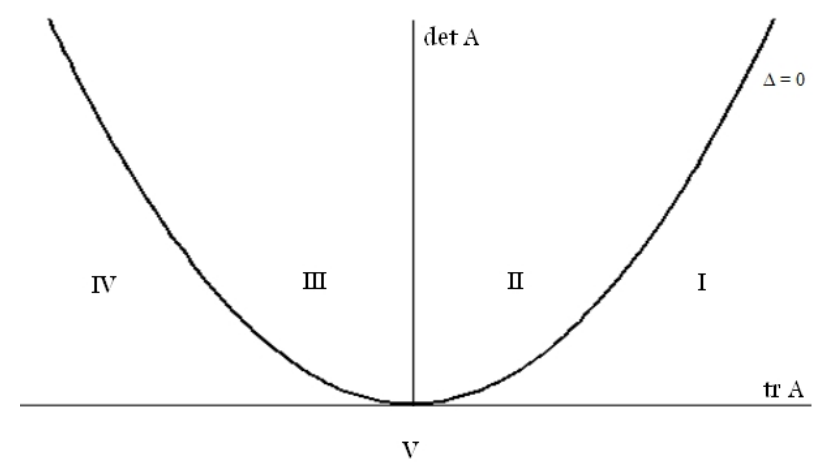

Figure 1: Linear stability

The above description of all possible cases in 2-dimension suggests that linear stability and Jacobi stability do not have to agree in all cases. In fact, the stability in Jacobi sense refers to a linear stability type of the trajectories in the curved space endowed with a nonlinear connection and a curvature tensor. Here the role of usual partial derivative is played by the covariant derivative along the flow. This suggests the difference in the meaning of linear stability and Jacobi stability. Nevertheless, in the present paper, we have found a known physical case, namely, the stability of non-degenerate fixed points in a Hamiltonian system with one degree of freedom, where these two stability concepts would match. 


\section{Hamiltonian Systems in the Plane}

Definition. A system of differential equations on $\mathbf{R}^{2}$ is said to be Hamiltonian with one degree of freedom if it can be expressed in the form

$$
\left\{\begin{array}{l}
\dot{x}=+\frac{\partial H}{\partial y} \\
\dot{y}=-\frac{\partial H}{\partial x}
\end{array}\right.
$$

where $H(x, y)$ is a twice-continuously differentiable function. Since from (19)

$$
\frac{d H}{d t}=\dot{x} \frac{\partial H}{\partial x}+\dot{y} \frac{\partial H}{\partial y}=\frac{\partial H}{\partial x} \cdot \frac{\partial H}{\partial y}-\frac{\partial H}{\partial y} \cdot \frac{\partial H}{\partial x} \equiv 0,
$$

which means $H(x, y)$ is constant along the solution curves of (19) (a first integral and a constant of motion), and the trajectories lie on the contours defined by $H(x, y)=C$, where $C$ is a constant. This is the conservation of total energy $H(x, y)$. The system is said to be conservative and there is no dissipation. In the next section we make comparative study of planar Hamiltonian systems from Liapunov and Jacobi viewpoints and assert that the results are compatible.

\section{Stability Analysis}

\subsection{Liapunov Stability Analysis}

We next establish some very specific results about the nature of the equilibrium points of Hamiltonian systems with one degree of freedom (19). Although Liapunov Stability analysis of planar Hamiltonian systems are known and textbook material [16] but we develop them here in details because this analysis will be used to establish Jacobi stability analysis. Note that the equilibrium points of the system (19) correspond to the critical points of the Hamiltonian function $H(x, y)$, where $\frac{\partial H}{\partial x}=\frac{\partial H}{\partial y}=0$ which are either relative extremas or a saddle. We may, without loss of generality, assume that the equilibrium point in question has been translated to the origin.

Lemma. If the origin is a stable (unstable) focus of the Hamiltonian system (19), then the origin is not a strict local minimum (maximum) of the Hamiltonian function $H(x, y)$.

Proof. Suppose that the origin is a stable focus, for instance. Then

$$
H\left(x_{0}, y_{0}\right)=\lim _{t \rightarrow \infty} H\left(x\left(t, x_{0}, y_{0}\right), y\left(t, x_{0}, y_{0}\right)\right)=H(0,0)
$$


for all $\left(x_{0}, y_{0}\right) \in N_{\epsilon}(0,0)$, where $N_{\epsilon}$ denotes a small deleted neighborhood of the origin. However, $H(x, y)>H(0,0)$ at a local minimum and $H(x, y)<H(0,0)$ at a local maximum, a contradiction. A similar argument can be applied when the origin is an unstable focus.

Recalling that (Theorem 2) if the equilibrium point is a center for the linearized planar system then the equilibrium point is either a center or a focus for the corresponding nonlinear system, we arrive at the following theorem:

Theorem 5. Any non-degenerate equilibrium point of the Hamiltonian system (19) is either a (topological) saddle or a center; furthermore $\left(x_{0}, y_{0}\right)$ is a (topological) saddle for (19) iff it is a saddle of the Hamiltonian function $H(x, y)$ and a strict local maximum or minimum of the function $H(x, y)$ is a center for (19).

Proof. We assume that the equilibrium point is at the origin. Thus, $H_{x}(0,0)=H_{y}(0,0)=0$ where $H_{x}$ and $H_{y}$ are partial derivatives of $H$ with respect to $x$ and $y$, respectively. The linearization of (19) at the origin is

$$
\dot{\mathbf{x}}=\mathbf{A x},
$$

where

$$
\mathbf{A}=\left[\begin{array}{ll}
H_{y x}(0,0) & H_{y y}(0,0) \\
-H_{x x}(0,0) & -H_{x y}(0,0)
\end{array}\right]
$$

is the Jacobian matrix at the equilibrium point $(0,0)$ for the system (19). We see that $\operatorname{tr} \mathbf{A}=0$ and that $\operatorname{det} \mathbf{A}=H_{x x}(0,0) H_{y y}(0,0)-H_{x y}^{2}(0,0)$. Thus, the equilibrium point at the origin is a saddle of the function $\mathrm{H}(\mathrm{x}, \mathrm{y})$ iff $\operatorname{det} \mathbf{A}<0$ (known as second partial derivative test) iff it is a saddle for the linear system (20) iff it is a (topological) saddle for the Hamiltonian system (19). Also, if $\operatorname{tr} \mathbf{A}=0$ and $\operatorname{det} \mathbf{A}>0$, the origin is a center for the linear system (20). And then, the origin is either a center or a focus for (19) according to Theorem 2. Thus, if the non-degenerate critical point $(0,0)$ is a strict local maximum or minimum of the function $H(x, y)$, then $\operatorname{det} \mathbf{A}>0$ and, according to the above lemma, the origin is not a focus for (19); i.e., the origin is a center for the Hamiltonian system (19). We must emphasis here that $\operatorname{det} \mathbf{A} \neq 0$ since the equilibrium points are assumed to be non-degenerate. We also recall that centers are always stable but not asymptotically stable. To summarize, the non-degenerate fixed point $(0,0)$ is

$$
\left\{\begin{array}{lll}
\text { Liapunov stable (center) } & \text { iff } & \operatorname{det} \mathbf{A}>0 \\
\text { Liapunov unstable (saddle) } & \text { iff } & \operatorname{det} \mathbf{A}<0 .
\end{array}\right.
$$




\subsection{Jacobi Stability Analysis}

Following the discussion in Section 3.2 and the relation (18), we are now prepared to perform Jacobi stability analysis for the vector field (19). Recalling

$$
\left.4 P_{1}^{1}\right|_{(0,0)}=\Delta:=\operatorname{tr} \mathbf{A}^{2}-4 \operatorname{det} \mathbf{A}= \begin{cases}<0 & \text { Jacobi Stable } \\ \geq 0 & \text { Jacobi Unstable }\end{cases}
$$

where $\mathbf{A}$ is the Jacobian matrix of the vector field at the fixed point given by (21). As stated above $\operatorname{tr} \mathbf{A}=0$ and $\operatorname{det} \mathbf{A}=H_{x x}(0,0) H_{y y}(0,0)-H_{x y}^{2}(0,0)$. Therefore we have

$$
\left.4 P_{1}^{1}\right|_{(0,0)}=\left.\Delta\right|_{(0,0}=0-4 \operatorname{det} \mathbf{A}=-4 \operatorname{det} \mathbf{A} .
$$

Therefore, the non-degenerate fixed point $(0,0)$ is

$$
\left\{\begin{array}{l}
\text { Jacobi stable iff } \quad \operatorname{det} \mathbf{A}>0 \\
\text { Jacobi unstable iff } \quad \operatorname{det} \mathbf{A}<0,
\end{array}\right.
$$

which is in complete agreement with the condition derived from Liapunov stability condition (22).

\section{Discussions and Final Remarks}

Two essentially different stability analysis methods, namely Liapunov stability analysis and the Jacobi stability analysis are reviewed in the present paper. Bohmer et al in [19] have considered the stability properties of several dynamical systems that play important roles in gravitation and cosmology where both methods of Liapunov linear stability analysis and Jacobi stability analysis, or the KCC theory, were used. Their study of stability has been done by analyzing the behavior of steady states of the respective dynamical system. Linear stability analysis is performed by the linearization of the dynamical system via the Jacobian matrix of a non-linear system at the fixed points, while the KCC theory involves stability of a whole trajectory in a tubular region [17], where Jacobi stability (or instability) means that the trajectories of the structure equations will bunch together (or disperse) when approaching the fixed point.

Boehmer et al in [19] have shown that there is a good correlation (but not perfect) between the linear stability of the fixed points, and the robustness of 
the corresponding trajectory to a small perturbation. In fact, there are cases where a fixed point is Liapunov stable but Jacobi unstable or vice versa. In the present paper, we have explicitly shown that (in)stability derived from Liapunov stability analysis is exactly the same as for Jacobi (in)stability for the case of a Hamiltonian system with one degree of freedom (planar).

\section{References}

[1] H. Abolghasem, Liapunov stability versus Jacobi stability, The Journal of Dynamical Systems and Geometric Theories, 10, No. 1 (2012), 13-32.

[2] H. Abolghasem, Jacobi stability of circular orbits in central forces, The Journal of Dynamical Systems and Geometric Theories, 10, No. 2 (2012), 197-214.

[3] H. Abolghasem, Stability of circular orbits in Schwarzschild spacetime, International Journal of Differential Equations and Applications, 12, No. 3 (2013), 131-147.

[4] P.L. Antonelli, Tensor N.S., 52 (1993), 27.

[5] P.L. Antonelli, Equivalence problem for systems of second-order ordinary differential equations, Encyclopedia of Mathematics, Kluwer Academic Publishers, Dordrecht (2000).

[6] P.L. Antonelli, I. Bucataru, New results about the geometric invariants in KCC-theory, An.St. Univ. "Al.I.Cuza" Iasi. Mat. N.S., 47 (2001), 405-420

[7] P.L. Antonelli (Editor), Handbook of Finsler Geometry, 1, Kluwer Academic, Dordrecht (2003).

[8] V.I. Arnold Mathematical Methods of Classical Mechanics, 2nd edition, Springer (1989)

[9] V. Balan, I.R. Nicola, Berwald-Moor metrics and structural stability of conformally-deformed geodesic SODE, Applied Sciences, 11 (2009), 19-34

[10] D. Bao, S.S. Chern, Z. Shen, An Introduction to Riemann-Finsler Geometry, Springer (2000). 
[11] C.G. Boehmer, T. Harko, Nonlinear stability analysis of the Emden-Fowler equation, ArXiv: 0902.1054v4 (2009).

[12] E. Cartan, Math. Z., 37 (1933), 619.

[13] S.S. Chern, Bulletin des Sciences Mathematiques, 63 (1939), 206.

[14] D.D. Kosambi, Math. Z., 37 (1933), 608.

[15] J. Hale, H. Kocak, Dynamics and Bifurcations, Springer-Verlag (1991).

[16] L. Perko, Differential Equations and Dynamical Systems, 3-rd Edition, Springer (2006).

[17] S.V. Sabau, Some remarks on Jacobi stability, Nonlinear Analysis, 63 (2005), 143.

[18] T. Harko, V.S. Sabau, Jacobi stability of the vacuum in the static spherically symmetric brane world models, Physical Review D, 77, No. 10 (2008), 104009.

[19] C.G. Boehmer, T. Harko, S.V. Sabau, Jacobi stability analysis of dynamical systems-applications in gravitation and cosmology, ArXiv: 1010.5464 (2010).

[20] R.M. Wald, General Relativity, University Of Chicago Press (1984).

[21] S. Wiggins, Introduction to Applied Nonlinear Dynamical Systems and Chaos, 3-rd Edition, Springer-Verlag (1997). 